\title{
XV. Die chemische Zusammensetzung und das specifische Gewicht des Sulfoborits.
}

Von

Konstantin Thaddéeff in Aachen.

Von den neu entdeckten Mineralen ist der interessantesten eines unzweifelbaft der Sulfoborit von Westeregeln. Die erste chemische Analyse dieses Minerals ruhrt von den Herren A. Naupert und W. Wen se ${ }^{1}$ ) her, welche die Schwefelsäure, die Magnesia und das Wasser auf directem Wege, die Borsäure dagegen aus der Differenz bestimmten. Da diese Analyse gegen die aufgestellte Formel in der Borsäure ein Fehlen von 1,88 \%, dagegen einen Ueberscbuss von 1,79\% im Wassergehalte ergab, so war eine Controlanalyse natürlich wünschenswerth. Herr Prof. Bücking in Strassburg, welchem wir eine vollständige krystallographiscbe Beschreibung des Sulfoborits verdanken, hatte daher, unter Aufwand grosser Muhe, $2 \mathrm{~g}$ des Minerals ausgesucht und sie mir, auf meine Bitte hin, zu Verfügung gestellt.

\section{Bestimmung des specifischen Gewichtes.}

Da der Sulfoborit beim Kochen mit Wasser zersetzt wird, so war ich gezwungen, sein specifisches Gewicht mit Hülfe des Pyknometers in Benzol zu bestimmen. Um aber für diese verhältnissmässig schwierige Bestimmung eine Controle zu haben, stellte ich Versuche mit der sogen. Schwebomethode an, welche dem verstorbenen Retgers ${ }^{2}$ ) so ausgezeichnete Resultate ergeben hatte. Das benutzte Pyknometer war genau desselben Baues wie dasjenige, welches ich bei den Bestimmungen der specifischen Gewichte der Olivine verwendete ${ }^{3}$ ).

1) Berichte der deutschen chem. Gesellschaft 1893, S. 874 ; Sitzungsberichte der Akademie d. Wissensch. zu Berlin 1893, S. 967. Ref. in dieser Zeitschr. 25, 621 u. 622.

2) Zeitschr. f. physik. Chem. 1889, 3, 497-561.

3) Diese Zeitschr. 1896, 26, 31. 


\section{a. Bestimmung des specifischen Gewichtes mit Hülfe de日 Methylenjodids.}

Zu diesem Zwecke bediente ich mich eines kleinen Trichters, dessen Ende mit einem Gummischlauche, an welchem ein Quetschhahn, versehen war. In den Trichter wurde Methylenjodid eingegossen, und ein durchsichtiger Krystall des Sulfoborits hineingebracht. Durch Zusatz von Benzol wurde das specifische Gewicht des Melhylenjodids soweit verringert, dass das Kryställchen entweder langsam sank oder langsam aufschwamm oder in der Flússigkeit ruhig schwebte. Sobald einer dieser Zustände abgepasst war, liess ich durch Oeffnen des Quetschhahnes die Flüssigkeit in das Pyknometer fliessen, schloss und trocknete dieses sorgfältig ab und wog es.

Das Gewicht des Pyknometers mit Wasser bei $20^{\circ} \mathrm{C}$. betrug im Mittel aus zehn Bestimmungen, deren Schwankungen höchstens $\pm 0,0005 \mathrm{~g}$ erreichten, 18,9700 g; das Gewicht des leeren Pyknometers 13,3833 g.

Da sich sowohl Methylenjodid, als auch Benzol rasch verfluchtigen, so konnte ich die Methode der genauen Wăgung, durch Schwingungsbeobachtungen bei verschiedenem Gewichte und Berechnung der Empfindlichkeit der Wage, nicht anwenden, sondern begnügte mich mit Schwingungsbeobachtungen bei einer und derselben Wägung, indem ich die Empfindlichkeit der Wage durch eine Reibe von Beobachtungen mit einem, dem mit Methylenjodid gefüllten Pyknomeler annähernd gleichen Gewichte bestimmte, um daraus das wahre Gewicht zu berechnen. Die Temperatur wurde nur in dem Augenblicke des Ablassens der Flussigkeit in das Pyknometer beobachtet. Jede Bestimmung wurde mehrere Male wiederholt. Zur Berechnung des specifischen Gewichtes bediente ich mich der in F. Koh Iraus ch's "Leitfaden der praktiscben Physik “, 7. Aufl. 1892, angegebenen Formeln. Die Ergebnisse sind folgende:

Krystall Nr. 1: Temp.: Gew. d. Pyknom. Spec. Gew. d. Mischung mit $\mathrm{CH}_{2} J_{2}+\mathrm{C}_{6} \mathrm{H}_{6}$ b.d.beob.Temp. bei $+4^{0}$ Mittel :

$\begin{array}{llllll}\text { 1) stieg auf } & 210 \mathrm{C} . & 27,1103 \mathrm{~g} & 2,458 & 2,451 & 2,449 \\ \text { 2) - - } & 21,4 & 27,0845 & 2,453 & 2,446 & \\ \text { 3) scbwebte } & 20,5 & 27,0997 & 2,459 & 2,453 & 2,450 \\ \text { 4) - } & 21,3 & 27,0873 & 2,454 & 2,447 & \\ \text { 5) sank } & 21,2 & 26,9824 & 2,434 & 2,427 & 2,428 \\ \text { 6) - } & 21,5 & 26,9815 & 2,436 & 2,429 & \end{array}$

Krystall Nr. 2.

\begin{tabular}{|c|c|c|c|c|c|}
\hline 1) & tieg auf & 21,8 & 27,1205 (?) & 2,460 & 2,453 \\
\hline 2) & - & 21 & 27,0612 & 2,449 & 2,443 \\
\hline 3) & - & 20,6 & 27,0653 & 2,449 & 2,443 \\
\hline 4) & - & 20,3 & 27,0764 & 2,451 & 2,445 \\
\hline
\end{tabular}


Gew. d. Pyknom.

Spec. Gew. d. Mischung

Temp.: mit $\mathrm{CH}_{2} J_{2}+\mathrm{C}_{6} \mathrm{H}_{6}$ b.d. beob. Temp. bei +40 Mittel:

5) schwebte $20,7^{\circ}$ C. $27,0563 \mathrm{~g}$

6) -

20,8

27,0843

2,448

20,4

27,0514

20

27,0359

2,452

2,442

7) sank

8) -

2,445

2,444

2,446

2,444

2,439

2,438

2,4385

Die Beobachtungen am Krystalle Nr. 2 weisen Schwankungen innerhalb viel engerer Grenzen (mit Ausnahme des Versuchs 1) auf, als beim Krystall Nr. 1 und dürften daher die genaueren sein. Man sieht auch, dass die Bestimmung des spec. Gewichtes des Flüssigkeitgemisches, in welchem das Kryställchen schwebte, infolge der raschen Verfluchtigung des Benzols nicht genau ist. Schon in der kurzen Zeit, während welcher die Flussigkeit in das Pyknometer abgelassen wurde, nahm ihr spec. Gewicht erheblich zu und erreichte jene Höbe, bei welcher das Kryställchen an die Oberfläche stieg. Aus diesem Grunde dürfte das Mittel aus den Beobachtungen an den Flüssigkeiten mit dem höchsten und niedrigsten spec. Gewichte der Wahrheit näher kommen.

Krystall Nr. 1 ergiebt bei $\frac{20,5^{0}-21,5^{0} \mathrm{C} .}{+4^{0} \mathrm{C} .}$ das spec. Gew. 2,438

$$
-\quad \text { Nr. } 2-\quad-\frac{20^{0}-21,8^{0} \mathrm{C} .}{+4^{0} \mathrm{C} .}-\quad-2,442
$$

oder im Mittel

$$
\frac{20^{0}-21,8^{0} \mathrm{C} .}{+4^{0} \mathrm{C} .}-\quad-2,440
$$

fur durchsichtige (frische) Sulfoboritkrystalle.

Fast denselben Werth ergaben mir Beobachtungen in demselben Methylenjodid mit Hulfe der Indicatoren von Peter Stoë (Heidelberg). Die klaren Sulfoboritkrystalle waren schwerer als Colemanit (2,422) und leichter als Leucit (2,468), was im Mittel zu 2,445 fuhrt.

Um das Material auf seine Homogenităt zu prüfen, wurden zweimal je zehn nicht ausgesuchte Krystalle genommen, und es wurde das spec. Gewicht jedes einzelnen Krystalles mit Hulfe derselben Indicatoren bestimmt. Von den ersten zehn Krystallen zeigte einer das spec. Gewicht 2,28, einer 2,33, drei 2,40, zwei 2,42 und zwei 2,445. Die anderen zehn Krystalle ergaben : für einen das spec. Gewicht 2,33, für zwei 2,40 und für sieben 2,445 .

Diese Versuche zeigen auf das Deutlichste, wie unhomogen die Substanz war.

Da es aber von Interesse war, das spec. Gewicht des zur Analyse zu verwendenden Materials zu bestimmen, so nahm ich die Bestimmung im Pyknometer vor, wobei stalt des Wassers, welches, wie schon bemerkt wurde, beim Kochen den Sulfoborit zersetzt, Benzol genommen wurde, 
b. Bestimmung des specifischen Gewichtes im Pyknometer mit Benzol.

Wie leicht es auch auf den ersten Blick erscheinen mag, das spec. Gewicht in Benzol zu bestimmen, lernt man doch recht bald die Unbequemlichkeiten dieses Verfahrens kennen.

Erstens verfluchtigt sich das Benzol sehr leicht, weshalb die Wägungen sehr schnell geschehen mussen unter Berucksichtigung derselben Vorsichtsmaassiegeln, welche auch beim Methylenjodid beobachtet werden mussten und oben (S. 265) erwähnt worden sind.

Zweitens verändern die geringsten Beimengungen von Wasser, Toluol etc. merklich das spec. Gewicht des Benzols. Daraus erklärt es sich denn auch, dass verschiedene Beobachter zu so verschiedenen Werthen fur das spec. Gewicht des Benzols gelangten. So fanden A drieenz bei $15^{\circ} \mathrm{C}$. 0,88462, $\mathrm{P}$ is ati und Paternò bei derselben Temperatur 0,883573, Janovsky 0,8872 und endlich Lachowicz bei $\frac{15^{0}}{+4^{0}}$ G. für »luftfreies « Benzol 0,87868 I). Ich musste also nothgedrungen das spec. Gewicht des von mir anzuwendenden Benzols bestimmen.

Nicht ausgekochtes Benzol gab mir folgende Werthe: Gewicht des leeren Pyknometers: 10,4499 g; Gewicht des mit Wasser gefüllten Pyknometers bei $20^{\circ} \mathrm{C}$.: $16,1550 \mathrm{~g}$ als Mittel aus 14 Beobachtungen, deren Minimum 16,1546 g und Maximum $16,1559 \mathrm{~g}$ war.

\begin{tabular}{|c|c|c|}
\hline $\begin{array}{l}\text { Temp.: } \\
19,5^{0} \mathrm{C} .\end{array}$ & $\begin{array}{c}\text { Pyknom. m. Benzol: } \\
15,4741 \mathrm{~g}\end{array}$ & $\begin{array}{c}\text { Spec. Gew. d. Benzols: } \\
0,88057\end{array}$ \\
\hline 19,7 & 15,4717 & 0,88018 \\
\hline 20,2 & 15,4684 & 0,87968 \\
\hline 20,5 & 15,4670 & 0,87948 \\
\hline 20,6 & $15,4661\}, 4654 \mathrm{~g}$ & 0.87929 \\
\hline 20,6 & $15,4648 f \cdots, 4004 \mathrm{~g}$ & 0,01022 \\
\hline 20,7 & 15,4654 & 0,87923 \\
\hline 20,8 & 15,4660 & 0,87935 \\
\hline 21,0 & 15,4626 & 0,87879 \\
\hline 21,1 & 15,4633 & 0,87893 \\
\hline 21,5 & 15,4617 & 0,87872 \\
\hline 21,8 & $15,4581 \backslash 15,4584 \mathrm{~g}$ & 087819 \\
\hline 21,8 & $15,4587\}$ & 0,01010 \\
\hline 21,9 & 15,4595 & 0,87840 \\
\hline 22,1 & 15,4572 & 0,87804 \\
\hline 22,4 & 15,4560 & 0,87788 \\
\hline 22,5 & 15,4555 & 0,87780 \\
\hline 23,4 & 45,4518 & 0,87732 \\
\hline
\end{tabular}

Wie man aus diesen Werthen leicht ersehen kann, war der Stöpsel meines Pyknometers nicht genau genug eingeschliffen, so dass die Schwankungen im Gewichte bei einer Temperaturdifferenz von $0,1^{0} \mathrm{C}$. bereits innerbalb der Fehlergrenzen lagen. Bei

1) Beilstein, Handb. d. organ, Chem., 3. Aufl., 1896, 2, 23, 
den folgenden Beobachtungen verwendete ich daher ein Pyknometer mit äusserst genau eingeschliffenem Stöpsel. Allein auch die vorstehenden Beobachtungen reichten aus, um sich zu überzeugen, wie erheblich der Einfluss der Temperatur ist. Beobachtungen bei constanter Temperatur sind aber wegen der Leichtflüchligkeit des Benzols undurchführbar. Anderentheils ist sein Ausdehnungscoëfficient unbekannt, und so musste ich denn für die vorzunehmenden Correctionen zur einfachen Interpolation greifen. Zu diesem Zwecke leitete ich zunächst aus allen Beobachtungen das Gewicht des Pyknomelers mit Benzol bei $20^{\circ} \mathrm{C}$. ab, indem ich berücksichtigte, dass bei Erhöhung der Temperatur um $10 \mathrm{C}$. das Gewicht des mit Benzol gefüllten Pyknometers um 0,005718 g abnimmt - bei $19,5^{0} \mathrm{C}$. ist es $15,4741 \mathrm{~g}$ und bei $23,4^{\circ} \mathrm{C}$. $15,4518 \mathrm{~g}$ schwer; demnach beträgt bei Zunahme um 3,90 C. die Gewichtsabnahme $0,0223 \mathrm{~g}$, oder für je $10 \mathrm{C}$. 0,005718 g. Meine achtzehn Versuche ergaben mir auf diese Weise auf $200 \mathrm{C}$. umgerechoet das Gewicht 15,4698 g, wobei das Maximum 15,4712 g und das Minimum 15,4683 g entsprach. Unter Zugrundelegung der beiden Werthe: Gewicht des Pyknometers mit Benzol bei $80^{\circ} \mathrm{C} .15,4698 \mathrm{~g}$ und Abnahme des Gewichtes um 0,005718 $\mathrm{g}$ bei einer Temperalurzunahme um je $10 \mathrm{C}$., erhält man folgende umgerechnete Werthe:

\begin{tabular}{lccc} 
& \multicolumn{3}{c}{ Gewicht des Pyknometers mit Benzol. } \\
Temp.: & Beobachtet: & Berechnet: & Differenz: \\
$19,5^{0} \mathrm{C}$. & $15,4741 \mathrm{~g}$ & $15,4727 \mathrm{~g}$ & $-0,0014 \mathrm{~g}$ \\
19,7 & 15,4717 & 15,4715 & $-0,0002$ \\
20,2 & 15,4684 & 15,4687 & $+0,0003$ \\
20,5 & 15,4670 & 15,4669 & $-0,0001$ \\
20,6 & 15,4654 & 15,4664 & $+0,0010$ \\
20,7 & 15,4654 & 15,4658 & $+0,0004$ \\
20,8 & 15,4660 & 15,4652 & $-0,0008$ \\
21,0 & 15,4626 & 15,4641 & $+0,0015$ \\
21,1 & 15,4633 & 15,4635 & $+0,0002$ \\
21,5 & 15,4617 & 15,4612 & $-0,0005$ \\
21,8 & 15,4584 & 15,4595 & $+0,0014$ \\
21,9 & 15,4595 & 15,4589 & $-0,0006$ \\
22,1 & 15,4572 & 15,4578 & $+0,0006$ \\
22,4 & 15,4560 & 15,4561 & $+0,0001$ \\
22,5 & 15,4555 & 15,4555 & 0, \\
23,4 & 15,4518 & 15,4504 & $-0,0014$
\end{tabular}

Die Schwankungen sind im positiven wie negativen Sinne gleich gross: in Summa $-0,0050 \mathrm{~g}+0,0052 \mathrm{~g}=+0,0002 \mathrm{~g}$ bei 16 Beobachtungen. Sie zeigen, dass diese Berechnungsweise der Wahrheit nahe kommende Werthe ergiebt, indem selbst die hochsten Abweichungen von $\pm 0,0014 \mathrm{~g}$ verhältnissmassig wenig das specifische Gewicht des Sulfoborits beeinflussen werden. Zur Berechnung des specifischen Gewichtes des Benzols wendete ich dasselbe Verfahren an:

Bei 200 C. wog das Pyknometer mit Benzol im Mittel 15,4698 g, woraus das spec. Gewicht des Benzols bei $\frac{+20^{\circ}}{+20^{\circ}}$ C. $=0,87989$ sein würde, da das Gewicht des Pyknometers mit Benzol bei 19,50 C. 15,4741 g und bei $23,4^{\circ} \mathrm{C}$. 15,4518 g betrug, und die entsprechenden specifischen Gewichte des Benzols 0,88057 bezw. 0,87732 sind, somit einer Temperaturdifferenz von $3,9^{\circ} \mathrm{C}$. eine Differenz im specifischen Gewicbte von 0,00325 odẹr von 0,000883 auf $10 \mathrm{C}$. entspricht, 
Die chemische Zusammensetzung und das specifische Gewicht des Sulfoborits. 269

\begin{tabular}{lccc} 
& \multicolumn{3}{c}{ Spec. Gewicht des Benzols. } \\
Temp.: & Beobachtet: & Berechnet: & Differenz: \\
$\mathbf{1 9 , 5 0}$ C. & 0,88057 & $\mathbf{0 , 8 8 0 3 0}$ & $-0,00027$ \\
19,7 & $\mathbf{0 , 8 8 0 4 8}$ & $\mathbf{0 , 8 8 0 1 4}$ & $-0,00004$ \\
20,2 & 0,87968 & $\mathbf{0 , 8 7 9 7 2}$ & $+0,00004$ \\
$\mathbf{2 0 , 5}$ & 0,87948 & $\mathbf{0 , 8 7 9 4 8}$ & 0 \\
20,6 & 0,87922 & 0,87939 & $+0,00017$ \\
20,7 & 0,87923 & 0,87931 & $+0,00008$ \\
20,8 & 0,87935 & 0,87922 & $-0,00013$ \\
21,9 & 0,87879 & 0,87906 & $+0,00027$ \\
21,1 & 0,87893 & 0,87897 & $+0,00004$ \\
21,5 & 0,87872 & 0,87864 & $-0,00008$ \\
21,8 & 0,87819 & 0,87839 & $+0,00020$ \\
21,9 & 0,87840 & 0,87831 & $-0,00009$ \\
22,1 & 0,87804 & 0,87814 & $+0,00010$ \\
22,4 & 0,87788 & 0,87789 & $+0,00001$ \\
22,5 & 0,87780 & 0,87781 & $+0,00001$ \\
23,4 & 0,87732 & 0,87706 & $-0,00026$
\end{tabular}

Ausgekochtes Benzol lieferte nachstehende Resultate: Gewicht des leeren Pyknomelers 13,3833 g; Gewicht des Pyknometers mit Wasser bei $20^{\circ} \mathrm{C}$. 18,9700 g, als Mittel aus zehn Bestimmungen mit dem Maximalwerthe 18,9705 g und dem Minimalwerthe $18,9693 \mathrm{~g}$.

Temp.: Pyknom. m. Benzol: Spec. Gew. des Benzols :

\begin{tabular}{|c|c|c|c|}
\hline $20,00 \mathrm{C}$. & $18,2970 \mathrm{~g}$ & & $\mathbf{0 , 8 7 9 5 3}$ \\
\hline 20,5 & 18,2944 & \multirow{2}{*}{$18,2945 \mathrm{~g}$} & \multirow{2}{*}{0,87916} \\
\hline 20,5 & 18,2946 & & \\
\hline 21,5 & 18,9894 & \multirow{3}{*}{18,28895} & \multirow{2}{*}{0,87834} \\
\hline 21,5 & 18,2885 & & \\
\hline 21,8 & 18,2870 & & 0,87804 \\
\hline 22,0 & 18,2859 & \multirow{4}{*}{18,2854} & \multirow{3}{*}{0,87780} \\
\hline 22,0 & 18,2853 & & \\
\hline 22,0 & 18,2851 & & \\
\hline $\mathbf{2 2 , 2}$ & 18,2849 & & 0,87775 \\
\hline 22,6 & 18,2825 & \multirow{5}{*}{18,2824} & \multirow{2}{*}{0,87738} \\
\hline 22,6 & 18,2823 & & \\
\hline 23,2 & $18,278.9$ & & 0,87684 \\
\hline 23,5 & 18,2767 & & 0,87650 \\
\hline 24,0 . & 18,2747 & & 0,87623 \\
\hline
\end{tabular}

Der Gewichtsunterschied des Pyknometers mit Benzol ist also innerhalb eines Temperalurintervalls von $4^{\circ} \mathrm{C}$. gleich $0,0223 \mathrm{~g}$; auf je $1^{0}$ würde demnach $0,005575 \mathrm{~g}$ Gewichtsabweichung kommen. Legt man diese Werthe zu Grunde, so kann man die für das Pyknometer mit Benzol für verschiedene Temperaturen gefundenen Gewichte auf $20^{\circ} \mathrm{C}$. umrechnen und erhält im Mittel 18,2970 g, bei einem Minimum von 18,2962 g und einem Maximum von $18,2973 \mathrm{~g}$, d. h. genau denselben Werth, welchen die Beobachtung ergab. Aus diesem Werthe nun und demjenigen, welcher die Gewichtsverminderung des mit Benzol gefüllten Pyknometers bei Erhbhung der Temperatur um $1^{\circ} \mathrm{C}$. angiebt, d. h. 0,005575 g, kann das Gewicht des Pyknometers mit Benzol bei den verschiedenen Temperaturen zurückberechnet werden. 


\begin{tabular}{lllc} 
& \multicolumn{3}{c}{ Gewicht des Pyknometers mit Benzol. } \\
Temp.: & Gefunden : & Berechnet : & Differenz : \\
$20,00 \mathrm{C}$. & $18,2970 \mathrm{~g}$ & $18,2970 \mathrm{~g}$ & 0 \\
20,5 & 18,2945 & 18,2942 & $-0,0003 \mathrm{~g}$ \\
21,5 & 18,28895 & 18,2887 & $-0,00025$ \\
21,8 & 18,2870 & 18,2870 & 0 \\
22,0 & 18,2854 & 18,2859 & $+0,0005$ \\
22,2 & 18,2849 & 18,2848 & $-0,0001$ \\
22,6 & 18,2824 & 18,2825 & $+0,0001$ \\
23,2 & 18,2789 & 18,2792 & $+0,0003$ \\
23,5 & 18,2767 & 18,2775 & $+0,0008$ \\
24,0 & 18,2747 & 18,2747 & 0
\end{tabular}

Da die Beobachtungen für das specifische Gewicht des ausgekochten Benzols bei $200 \mathrm{C}$. $0,87953 \mathrm{~g}$ und bei $24^{\circ} \mathrm{C} .0,87623 \mathrm{~g}$ ergaben, so folgt daraus bei einer Temperaturzunahme von $40 \mathrm{C}$. eine Verminderung des specifischen Gewichtes um 0,00330 oder bei einer solchen von $1^{0} \mathrm{C}$. um 0,000825. Diese Werthe gestatten nun eine Berechnung des specifischen Gewichtes des Benzols für verschiedene Temperaturen.

Spec. Gewicht des Benzols.

$\begin{array}{lccc}\text { Temp.: } & \text { Gefunden: } & \text { Berechnet: } & \text { Differenz: } \\ 20,00 \mathrm{C} . & \mathbf{0 , 8 7 9 5 3} & 0,87953 & 0 \\ \mathbf{2 0 , 5} & 0,87916 & \mathbf{0 , 8 7 9 1 2} & -0,00004 \\ \mathbf{2 1 , 5} & \mathbf{0 , 8 7 8 3 4} & \mathbf{0 , 8 7 8 2 9} & -\mathbf{0 , 0 0 0 0 5} \\ \mathbf{2 1 , 8} & 0,87804 & \mathbf{0 , 8 7 8 0 5} & +0,00004 \\ 22,0 & 0,87780 & \mathbf{0 , 8 7 7 8 8} & +0,00008 \\ 22,2 & 0,87775 & \mathbf{0 , 8 7 7 7 2} & -0,00003 \\ \mathbf{2 2 , 6} & \mathbf{0 , 8 7 7 3 8} & \mathbf{0 , 8 7 7 3 9} & +0,00004 \\ \mathbf{2 3 , 2} & 0,87684 & \mathbf{0 , 8 7 6 8 9} & +0,00005 \\ \mathbf{2 3 , 5} & 0,87650 & 0,87664 & +0,00014 \\ \mathbf{2 4 , 0} & 0,87623 & 0,87623 & 0\end{array}$

Der Fehler ist so gering, dass ich bei der Bestimmung des specifischen Gewichtes des Sulfoborits getrost diese Berechnungsweise in Anwendung bringen konnte.

Es verdient hervorgehoben zu werden, dass aus meinen Versuchen für das a us gekochte Benzol ein geringeres specifisches Gewicht hervorgeht, als für das nicht a u sge k o chte. Die beiden einander entsprechenden Werthe für $20^{\circ} \mathrm{C}$. sind: 0,87953 bezw. 0,87989. Wie ist dieser Widerspruch, dass das durch Auskochen "luftfrei " werdende Benzol statt schwerer zu werden leichter wird, zu erklären?

Herrn Dr. Ra u, Privatdocenten an der hiesigen Hochschule, verdanke ich hierüber die wichtige Aufklärung, dass es nicht die Luft ist, sondern das im käuflichen Benzol stets in kleinen Mengen enthaltene Wasser, welches diese Erscheinung bedingt. Dieses Wasser entweicht beim Destilliren des Benzols gleich mit dessen ersten Fractionen, weshalb auch das specifische Gewicht des zurückbleibenden, nunmehr wasserfreien Benzols erniedrigt wird. Dieser Umstand und die Befürchtung, dass der Sulfoboril auch beim Kochen in Benzol sich theilweise zersetzen könnte, veranlassten mich, das Benzol nicht noch einmal mit dem Sulfoborit zusammen auszukochen, sondern die noch etwa anhaftende Luft durch längeres Umrühren des Sulfoborits im Pyknometer mit einem Platindrahte zu vertreiben.

Die Ergebnisse der Beobachtungen waren folgende: Gewicht des leeren Pyknometers 13,3833 g; Gewicht des mit Wasser gefüllten Pyknometers bei 200 C. 18,9700 g; 
Die chemische Zusammensetzung und das specifische Gewicht des Sulfoborits. 271

Gewicht des Pyknometers mit a u g e k o chtem Benzol bei $20^{\circ}$ C. 18,2970 g; Verminderung dieses Gewichtes bei Zunahme der Temperatur un $1^{0} \mathrm{C}$. $0,005575 \mathrm{~g}$; Verminderung des specifischen Gewichtes des Benzols mit Zunahme der Temperatur um ${ }^{0} \mathrm{C}$. 0,000825 .

\begin{tabular}{|c|c|c|c|c|c|}
\hline \multicolumn{2}{|c|}{$\begin{array}{l}\text { Abgewogen } \\
\text { Sulfoborit : }\end{array}$} & Temp.: & $\begin{array}{l}\text { Gew. d. Pyknom. } \\
\text { m. Benzol u. Sulfoborit }\end{array}$ & $\begin{array}{l}\text { Gew. d. Pykn. } \\
\text { : m. Benzol: }\end{array}$ & $\begin{array}{l}\text { Spec. Gew. } \\
\text { d. Benzols }\end{array}$ \\
\hline 1) & $1,9991 \mathrm{~g}$ & $22,00 \mathrm{C}$. & $19,5610 \mathrm{~g}$ & $18,2859 \mathrm{~g}$ & 0,87788 \\
\hline 2) & - & 22,7 & 19,5583 & 48,2820 & 0,87730 \\
\hline 3) & - & 24,7 & 19,5488 & 18,2708 & 0,87565 \\
\hline 4) & - & 24,3 & 19,5510 & 18,2730 & 0,87598 \\
\hline 5) & - & 24,5 & 19,5505 & 18,2719 & 0,87582 \\
\hline & 1,9450 & 22,0 & 19,5257 & 18,2859 & 0,87788 \\
\hline 7) & - & 22,3 & 19,5248 & 18,2842 & 0,87763 \\
\hline 8) & - & 22,6 & 19,5225 & $18,282 \mathrm{z}$ & 0,87739 \\
\hline 9) & - & 23,0 & 19,5201 & 18,2803 & 0,87706 \\
\hline 10) & - & 22,0 & 19,5264 & 18,2859 & 0,87788 \\
\hline 11) & 1,0032 & 24,0 & 18,9128 & 18,2747 & 0,87623 \\
\hline 12) & - & 21,7 & 18,9266 & $18,287 \mathrm{~s}$ & 0,87813 \\
\hline 13) & - & 22,0 & 18,9262 & 18,2859 & 0,87788 \\
\hline 14) & - & 21,5 & 18,9282 & 18,2886 & 0,87829 \\
\hline 15) & - & 20,9 & 18,9324 & 18,2920 & 0,87879 \\
\hline
\end{tabular}

Auf Grund dieser Werthe und unter Zuhulfenahme der bekannten Formeln konnte nun das spec. Gewicht des Sulfoborits berechnet und darauf auf die Temperatur von $4^{\circ} \mathrm{C}$. umgerechnet werden.

\section{Speciflsches Gewicht des Sulfoborits.}

Temp. $10 \mathrm{C}$.

Temp. $+40 \mathrm{C}$.

$\left.\begin{array}{llll}\text { 1) } & 2,424 & 220 \mathrm{C} . & 2,417 \\ \text { 2) } & 2,427 & 22,7 & 2,420 \\ 3) & 2,427 & 24,7 & 2,418 \\ \text { 4) } & 2,428 & 24,3 & 2,420 \\ \text { 5) } & 2,430 & 24,5 & 2,422\end{array}\right\}$ Millel 2,4194.

Spec. Gew. Temp. Spec. Gew. Temp.

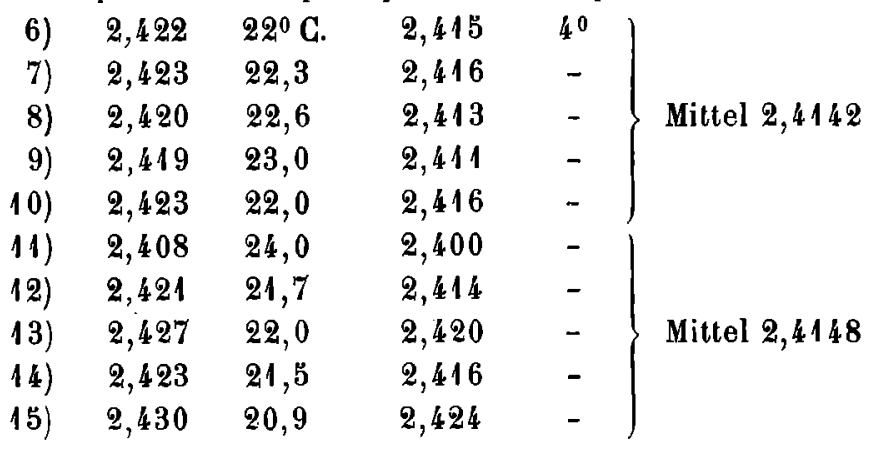


Im Mittel war also das spec. Gewicht des zur Analyse verwendeten Sulfoborits bei $\frac{20,9^{0}-24,7^{0}}{+4^{0}}$ C. 2,416, welches auf eine beginnende Zersetzung hinweist, da klare Krystalle im Mittel 2,440 ergaben.

A. Naupert und W. Wense hatten, nach dem von ihnen bestimmten spec. Gewichte von 2,38-2,45 oder im Mittel von 2,415 zu urtheilen, auch kein frisches Material zu ihrer Verfugung. Ihr Millelwerth 2,415 stimmt beinahe mit dem von mir gefundenen 2,416 uberein.

\section{Analyse des Sulfoborits.}

A. Naupert und W. Wense fanden im Sulfoborit die Bestandtheile $\mathrm{MgO}, \mathrm{SO}_{3}, \mathrm{~B}_{2} \mathrm{O}_{3}$ und $\mathrm{H}_{2} \mathrm{O}$. Da mir nicht viel Material zur Verfugung sland, so hielt ich es nicht für nöthig, die qualitative Prufung zu wiederholen, sondern ging direct zur quantitativen Analyse uber. Ausser den erwähnten Bestandtheilen bestimmte ich noch $\mathrm{Fe}_{2} \mathrm{O}_{3}$, da viele von den Kryställchen eine röthliche Färbung besassen. - Vorversuche mit Borax und Magnesiumsulfat zeigten, dass es unmöglich ist, die Borsäure und die Schwefelsäure in einer und derselben Portion zu bestimmen; es mussten daher zwei verschiedene abgewogene Mengen angewendet werden. Zur Bestimmung des Wassers bediente ich mich zunächst des Verfahrens von Si pöcz ${ }^{1}$ ), jedoch ohne Erfolg. Abgesehen von den an die Bestimmung selbst sich knupfenden Schwierigkeiten, hat diese Methode den Fehler, dass, wenn basische Salze mit Kalium- und Natriumcarbonat geschmolzen werden, ein Theil des Wassers in der Schmelze zuritckbleibt, da sich Aetzalkalien bilden können. In der That, wenn man z. B. $\mathrm{Ca}(\mathrm{OH})_{2}$ mit $\mathrm{Na}_{2} \mathrm{CO}_{3}$ schmilzt, so kann man sicher sein, dass die Reaction zum Theil nach der Formel

$$
\mathrm{Ca}(\mathrm{OH})_{2}+\mathrm{Na}_{2} \mathrm{CO}_{3}=\mathrm{CaCO}_{3}+2 \mathrm{NaOH}
$$

verlaufen wird. Dass aber durch einfaches Schmelzen aus $\mathrm{NaOH}$ das Wasser schwer auszulreiben ist, ist hinreichend bekannt. Daher ergab die Wasserbestimmung nach Sipöcz's Verfahren beim Sulfoborit, in welchem das Wasser kein Krystallwasser, sondern chemisch gebundenes ist, zu geringe Werthe. Später, bei Analysen basischer Kupfersalze, leitete ich beim Beginn des Schmelzens einen Kohlensäurestrom ein, wodurch das sich bildende $\mathrm{NaOH}$ wiederum in $\mathrm{Na}_{2} \mathrm{CO}_{3}$ ubergeführt wurde, und gelangle dabei zu recht befriedigenden Ergebnissen; der Gedanke, einen Kohlensäurestrom bei den Wasserbestimmungen im Sulfoborit anzuwenden, kam mir leider zu spăt, als diese Bestimmungen schon beendet waren. Für genauer balte ich die Bestimmung durch einfaches Gluhen (Analyse IV), obwohl ich auch die nach Sipöcz's Methode gefundenen Werthe (Analysen II und III) anfuhre.

1) Zeitschr. f. analytische Chemie 1878, 17, 206. 
Die chemische Zusammenselzung und das specifische Gewicht des Sulfoborits. 273

I. $0,5548 \mathrm{~g}$ fein gepulverten Sulfoborits ergaben nach einem von mir ausgearbeiteten Verfahren 1) - Ablreiben der Borsäure als $\left(\mathrm{CH}_{3}\right)_{3} \mathrm{BO}_{3}$ und Bestimmung als $\mathrm{KBFl}_{\mathbf{d}}$ $-0,4022 \mathrm{~g} \mathrm{KBFl}_{4}$ bezw. 0,11105 $\mathrm{B}_{2} \mathrm{O}_{3}$ entsprechend 20,01\%. Die im Kölbchen nach Abdestilliren der Borsäure zurückgebliebene Flüssigkeit enthielt einen in Schwefelsäure unlöslichen Rückstand von 0,0025 g entsprechend $0,45 \%$.

II. $0,2974 \mathrm{~g}$ Substanz zeigten nach 24 stïndigem Trocknen bei $160^{\circ}-170^{0}$ einen Verlust von $0,0001 \mathrm{~g}$ bezw. $0,03 \%$; mit $1,5 \mathrm{~g} \mathrm{KNaCO}_{3}$ nach $\mathrm{Sipöcz's} \mathrm{Methode} \mathrm{ge-}$ schmolzen, lieferten sie $0,0579 \mathrm{~g}$ bezw. $19,47 \% \mathrm{H}_{2} \mathrm{O}$. Die Schmelze wurde in das Kölb-

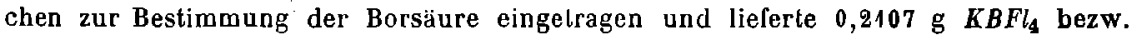
0,05818 g oder $19,56 \% B_{2} O_{3}$.

III. $0,5 \mathrm{~g}$ Substanz verloren nach 24 stündigem Trocknen bei $110^{0} 0,0003 \mathrm{~g}$ entsprechend $0,06 \%$; dann gaben sie mit $3 \mathrm{~g} \mathrm{KNaCO}$ geschmolzen $0,1052 \mathrm{~g}$ bezw. 21,04\% $\mathrm{H}_{2} \mathrm{O}$. In dieser Probe wurde nur noch die Magnesia bestimmt und 0,4666 $\mathrm{g}$ $\mathrm{Mg}_{2} \mathrm{P}_{2} \mathrm{O}_{7}$ entsprechend $0,1681 \mathrm{~g}$ oder $33,62 \% \mathrm{MgO}$ gefunden.

IV. $0,2526 \mathrm{~g}$ Substanz wurden in einem Platintiegel abgewogen und verloren nach 24 stïndigem Trocknen bei $1300-15000,0005 \mathrm{~g}$ entsprechend $0,20 \%$, dann nach sechsstündigem Glühen bei Rothglühhitze weitere $0,0592 \mathrm{~g}$ entsprechend $23,43 \%$. Nach Behandlung des Rückstandes mit $\mathrm{HCl}$ blieben ungelöst 0,0008 $\mathrm{g}$ entsprechend 0,34\%. Der Auflobsung wurde $\mathrm{BaCl}_{2}$ zugesetzt, welches $0,1633 \mathrm{~g} \mathrm{BaSO}_{4}$ fällle, entsprechend $0,05605 \mathrm{~g}$ oder 22,19\% $\mathrm{SO}_{3}$. Im Filtrate wurde das iuberschüssige $\mathrm{BaCl}_{2}$ mit $\mathrm{H}_{2} \mathrm{SO}_{4}$ niedergeschlagen und abfiltrirt; in der eingeengten Lösung wurde mit Ammoniak $\mathrm{Fe}_{2} \mathrm{O}_{3}$ gefällt und ergab $0,0002 \mathrm{~g}$ entsprechend $0,08 \%$; im Filtrate des $\mathrm{Fe}_{2} \mathrm{O}_{3}$ wurde endlich die Magnesia als Ammonium-Magnesium-Phosphat gefällt und lieferte $0,2350 \mathrm{~g} \mathrm{Mg}_{2} \mathrm{P}_{2} \mathrm{O}_{7}$ entsprechend $0,08465 \mathrm{~g}$ oder $33,51 \% \mathrm{MgO}$.

V. 0,2 g Substanz hinterliessen nach Auflösung in $\mathrm{HCl}$ einen unlöslichen Bückstand von $0,0004 \mathrm{~g}$ oder $0,20 \%$. Die Lösung wurde in gleicher Weise, wie bei der Anal. IV behandelt und lieferte: $0,1325 \mathrm{~g} \mathrm{BaSO}_{4}$ entsprechend $0,04547 \mathrm{~g}$ oder $22,73 \% \mathrm{SO}_{3}$; $0,0003 \mathrm{~g}$ oder $0,15 \% \mathrm{Fe}_{2} \mathrm{O}_{3} ; 0,1849 \mathrm{~g} \mathrm{Mg}_{2} \mathrm{P}_{2} \mathrm{O}_{7}$ entsprechend $0,0666 \mathrm{~g}$ oder $33,30 \% \mathrm{MgO}$

Stellen wir die Ergebnisse dieser fünf Analysen zusammen, so erhalten wir:

I. II. III. IV. V. Mittel :

\begin{tabular}{|c|c|c|c|c|c|c|}
\hline$M g O$ & - & - & 33,62 & 33,51 & 33,30 & 33,48 \\
\hline $\mathrm{SO}_{3}$ & - & 一 & - & 22,19 & 22,73 & 22,46 \\
\hline$B_{2} O_{3}$ & 20,01 & 19,56 & 一 & - & - & 19,79 \\
\hline $\mathrm{Fe}_{2} \mathrm{O}_{3}$ & - & - & 一 & 0,08 & 0,15 & 0,11 \\
\hline Glühverlust & - & - & - & 23,43 & $\cdots$ & 23,43 \\
\hline $\begin{array}{l}\text { Verlust beim Trock- } \\
\text { nen bei } 110^{\circ}-170^{\circ}\end{array}$ & - & 0,03 & 0,06 & 0,20 & - & 0,10 \\
\hline \multirow[t]{2}{*}{ Unlöslich } & 0,45 & - & - & 0,31 & 0,20 & $\mathbf{0 , 3 2}$ \\
\hline & & & & & & 99,69 \\
\hline
\end{tabular}

Die Analyse von Na upert und We se gab hingegen folgende Resultate:

$\mathrm{MgO} \quad 32,91$

$\mathrm{SO}_{3} \quad 21,95$ als Mittel aus zwei Bestimmungen 21,84 und 22,07

$\mathrm{H}_{2} \mathrm{O} \quad 21,50$

$\mathrm{B}_{2} \mathrm{O}_{3} \frac{(23,64)}{100,00}$ aus der Differenz zu 100

1) Die Arbeit erscheint in der Zeitschr. f. analyt. Chemie 1897.

Groth, Zeitsehrift f. Krystallogr. XXVIII. 
Dabei blieben als in Mineralsäuren unlöslicher Rückstand 0,4 bis 0,8\%. Auf Grund dieser Analyse wurde für den Sulfoborit die Formel

$$
3 \mathrm{MgSO}_{4}, 2 \mathrm{Mg}_{3} \mathrm{~B}_{4} \mathrm{O}_{9}, 12 \mathrm{HI}_{2} \mathrm{O}
$$

aufgestellt. $\mathrm{Zu}$ welcher Formel führen nun meine Analysen? Vernachlässigt man das $\mathrm{Fe}_{2} \mathrm{O}_{3}$ und den unlöslichen Rückstand, so hat man folgende Werthe, welche durch Division mit den Molekulargewichten das nebenstehende Molekularverbältniss ergeben:

in Proc.: Mol.-Gew.: Molekularverhältniss:

$\begin{array}{llllll}\mathrm{MgO} & 33,48 & 39,90 & 0,839 & 2,98 & 3 \\ \mathrm{SO}_{3} & 22,46 & 79,86 & 0,281 & 1 & 1 \\ \mathrm{~B}_{2} \mathrm{O}_{3} & 19,79 & 69,68 & 0,284 & 1,01 & 1 \\ \mathrm{H}_{2} \mathrm{O} & 23,53 & 17,96 & 1,310 & 4,66 & 4,5-5\end{array}$

Es ist ersichtlich, dass im Sulfoborit ein sehr einfach zusammengesetztes Doppelsalz $\mathrm{MgSO}_{4}, \mathrm{Mg}_{2} \mathrm{~B}_{2} \mathrm{O}_{5}$ vorliegt. Zweifelhaft erscheint nur der Wassergehalt. Meine directen Bestimmungen nach Sip ö z's Verfahren ergaben von einander sehr abweichende Werthe: 21,04 bezw. 19,56\%, von denen der erste demjenigen von $\mathrm{N}$ a upert und Wense, welche $21,50 \%$ fanden, sehr nahe kommt. Dass das Wasser im Sulfoborit als Constitutionswasser enthalten ist, darauf weisen meine Versuche mit dem Trocknen der Substanz bei $160^{\circ}-170^{\circ}$ hin, bei denen der Verlust höchstens $0,2 \%$ erreichte. Darum halte ich die Bestimmung durch Gluhen bei schwacher Rothgluth, welche im Ganzen 23,53\% ergab, für richtiger, zumal es schwer ist, sich vorzustellen, dass bei solchem Glühen Schwefelsäure- oder Borsäure-Anhydrid verloren gehen könnten und dies um so weniger, als beim Berechnen des Wassers aus der Differenz ein dem gefundenen nahe kommender Werth von $23,84 \%$ erhalten worden wäre.

Leider war das zur Analyse verwendete Material, wie es dessen spec. Gewicht zeigte, zum Theil zersetzt. Es fragt sich nur, welchen Verlauf mochte die Zersetzung nehmen? Verwittern, sein Krystallwasser verlieren konnte der Sulfoborit nicht, da er selbst beim Erwärmen auf $160^{\circ}-170^{\circ} \mathrm{C}$. höchstens $0,2 \%$ einbüsste; eher konnte er Wasser aufgenommen haben. Ausserdem verliert das Mineral beim Behandeln mit Wasser ziemlich leicht einen Theil des Magnesiumsulfats ${ }^{1}$ ), während sein Vorkommen in den Garnallit-Lösungsrückständen, sowie seine Grewinnungsweise aus diesen Rückständen, die Möglichkeit des Zerfalls der frischen Sulfoboritsubstanz in der angedeuteten Weise durchaus nicht ausschliessen. Rechnet man in der That die erhaltenen Mengen $\mathrm{MgO}, \mathrm{SO}_{3}, \mathrm{~B}_{2} \mathrm{O}_{3}$ und $\mathrm{H}_{2} \mathrm{O}$ (zusammen 99,26\%) auf $100 \mathrm{um}$, so erhält man die von der Formel $\mathrm{MgSO}_{4}, \mathrm{Mg}_{2} \mathrm{~B}_{2} \mathrm{O}_{5}, 4 \frac{1}{2} \mathrm{H}_{2} \mathrm{O}$ geforderten, sebr nahen Werthe:

1) Privalmiltheilung des Hrn. W. Wense an Hrn. Prof. Bü cking. 
Die chemische Zusammensetzung und das specifische Gewicht des Sulfoborits. 275

$\begin{array}{lccc} & \text { Gefunden: } & \text { Berechnet: } & \text { Differenz: } \\ \mathrm{MgO}_{\mathrm{O}} & 33,73 & 34,19 & -0,46 \\ \mathrm{SO}_{3} & 22,62 & 22,81 & -0,19 \\ \mathrm{~B}_{2} \mathrm{O}_{3} & 19,93 & \mathbf{1 9 , 9 0} & +0,03 \\ \mathrm{H}_{2} \mathrm{O} & \frac{23,70}{23,09} & +0,61 \\ & \frac{29,98}{99,99} & \end{array}$

Die Analyse spricht dafür, dass die Substanz beiden obenerwähnten Einflüssen ausgesetzt gewesen ist, und dass die Zusammensetzung des Sulfoborits durch die Formel

$$
\mathrm{MgSO}_{4}, \mathrm{Mg}_{2} \mathrm{~B}_{2} \mathrm{O}_{5}, 4 \frac{1}{2} \mathrm{H}_{2} \mathrm{O} \text { oder } 2 \mathrm{MgSO}_{4}, 2 \mathrm{Mg}_{2} \mathrm{~B}_{2} \mathrm{O}_{5}, 9 \mathrm{H}_{2} \mathrm{O}
$$

ausgedruckt werden kann. Von der Borsäure $\mathrm{H}_{3} \mathrm{BO}_{3}$ ausgehend kann man dieser Formel auch die Gestalt

geben.

$$
4 \mathrm{MgHBO}_{3}, 2 \mathrm{MgSO}_{4}, 7 \mathrm{H}_{2} \mathrm{O}
$$

Sehr zu bedauern ist es, dass der Sulfoborit so selten ist, und es ist daher nicht zu erwarten, dass es bald gelingen wird, neue Versuche zur Bestimmung seines Wassergehaltes anzustellen; in den Bestimmungen von $\mathrm{MgO}, \mathrm{SO}_{3}$ und $\mathrm{B}_{2} \mathrm{O}_{3}$ glaube ich dagegen kaum einen groben Fehler gemacht zu haben, da die Analyse so einfache Beziehungen zwischen diesen drei

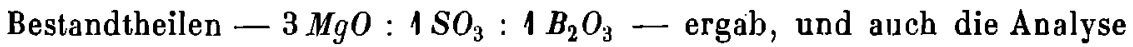
von $\mathrm{Na}$ upert und Wense zwischen $\mathrm{MgO}$ und $\mathrm{SO}_{3}$ dasselbe Verbältniss $3: 1$ feststellte.

Meine Bemuhungen, den Sulfoborit kunstlich darzustellen durch Erwärmen eines Gemisches von Magnesiumsulfat und Borax in Gegenwart von Wasser oder einer Kaliumchloridlösung bei $150^{\circ}-160^{\circ}$ in zugeschmolzenen Röhren, waren leider erfolglos. Ich erhielt dabei Magnesiumborate, jedoch ohne Betheiligung der Schwefelsäure, von der in der erhaltenen krystallinischen Masse höchstens nur Spuren nachgewiesen werden konnten.

Mineralogisches Institut der königl. techn. Hochschule,

14. März 1897. 